\title{
Ocorrência e Distribuição de Coccinelídeos (Coleoptera, Coccinellidae) Associados às Plantas Cítricas no Estado do Rio de Janeiro ${ }^{1}$
}

\author{
William Costa Rodrigues², Paulo Cesar Rodrigues Cassino ${ }^{3} \&$ Reinildes Silva Filho ${ }^{4}$
}

1. Parte da Dissertação de mestrado do primeiro autor. Projeto financiado pelo CNPq. 2. Universidade Severino Sombra e FAETEC/Instituto Superior de Tecnologia, Paracambi, RJ, e-mail: wcrodrigues@ebras.bio.br, Autor para correspondência. 3. Universidade Federal Rural do Rio de Janeiro, Bolsista de Produtividade do CNPq, e-mail: pr.cassino@uol.com.br. 4. Pós-Doutorado na Universidade Federal de Viçosa, e-mail: rennefilho@gmail.com

\section{EntomoBrasilis 1(2): 23-27 (2008)}

\begin{abstract}
Resumo. O estudo objetivou registrar a ocorrência, flutuação populacional das espécies de coccinelídeos e delinear a distribuição geográfica (Coleoptera, Coccinellidae) associados as plantas cítricas no Estado do Rio de Janeiro. Foram visitados 11 municípios situados em duas regiões do Estado do Rio de Janeiro: Baixada Fluminense e Região das Baixadas Litorâneas (Região Citrícola). O período de estudo foi de janeiro de 2000 a junho de 2001. Os coccinelídeos foram observados, coletados, conservados e identificados. Desta forma 11 espécies foram observadas no Estado. Os municípios com maior ocorrência de espécies foi Seropédica e Araruama e com menor ocorrência foram São Pedro da Aldeia, Iguaba Grande, Tanguá e Itaboraí. A espécie que se verificou com maior distribuição foi Pentilia egena (Mulsant, 1850), presente em 10 municípios, e com menor distribuição foi Curinus coeruleus Mulsant 1850, presente em apenas um município. Quando avaliada a flutuação populacional, houve uma tendência na redução da população nos meses mais frios (inverno), sendo P. egena a espécie com maior média populacional (40\%) e espécies dominantes juntamente com Azya luteipes Mulsant 1850. No período de estudo a diversidade de espécies foi considerada relativamente alta $(\alpha=3,91)$ quando avaliada através do índice de Margalef e Shanon-Wiener $\left(H^{\prime}=0,81\right)$, sendo considerada uma diversidade relativamente alta.
\end{abstract}

Palavras-Chave: Citros, controle biológico, inimigos naturais, predador

\section{Occurrence and Distribution of Coccinelids (Coleoptera, Coccinellidae) Associates at the Citrus Plants in Rio de Janeiro State}

Abstract. This work aimed to register the occurrence, study the population fluctuation the ladybeetles species and to delineate the geographic distribution (Coleoptera, Coccinellidae) associated at citrus plants in Rio de Janeiro State. 11 districts situated in two regions of the Rio de Janeiro State were visited: "Baixada Fluminense" and "Região das Baixadas Litorâneas (Região Citrícola)". The study period was January 2000 to June 2001. The coccinellids was observed, collected, conserved, and identified. This way 11 species they were observed on State. The districts to larger species occurrence were Seropédica, and Araruama and to species lower was São Pedro da Aldeia, Iguaba Grande, Tanguá, Itaboraí, Natividade e Porciúncula. The species that it was verified in larger distribution were Pentilia egena (Mulsant, 1850), present in 10 districts, and with smaller distribution it was Curinus coeruleus Mulsant 1850, present in just only one district. When evaluated population fluctuation, there was a tendency in the reduction of the population in coldest (winter) moths, being $P$. egena the specie with average larger populational (40\%) and dominant specie together with Azya luteipes Mulsant 1850. In study period the species diversity was high $(\alpha=3.91)$ when evaluated through Margalef and Shanon-Wiener index's $\left(H^{\prime}=0.81\right)$, being considered a relatively high diversity.

Key words: Biological control, citrus, natural enemies, predator

$\mathbf{O}$ registro de coccinelídeos predando várias espécies de homópteros tem sido relatado por vários autores (ARAúso 1940; Costa Lima 1941; Silva et al. 1968; Peracchi 1971; Bartoszeck 1976; Arioli \& LinK 1987a; CASSINo \& Rodrigues 2004), onde são vinculados ao controle biológico, muito mais que qualquer outro táxon de organismo predador, sendo importantes inimigos naturais de aleirodídeos, cochonilhas e afídeos (ОвRускі \& Kring 1998).

O número de espécies de coccinelídeos aproxima-se de 4200 , onde a maioria (90\%) são predadores benéficos, outros são fitófagos ou alimentam-se de fungos, sendo o hábito alimentar das larvas e adultos semelhantes. No caso dos afidófagos, a alimentação pode ser influenciada pela ocorrência de surtos de afídeos (e.g), do tipo de planta infestada e do comportamento dos coccinelídeos, que são na sua maioria polífagos (IPERTI 1999), entretanto freqüentemente demonstram preferência por uma determinada presa (Hoder 1973; Slogget \& MAJERus 2000).

Nos casos históricos de controle biológico, verifica-se que mais de 50 espécies de coccinelídeos estão associadas ao controle de aleirodídeos (Katsoyannos et al. 1997; ОвRYCKI \& KRING 1998; Ulusoy et al. 2003), cochonilhas (ARAújo 1940; Costa Lima 1941; Costa Lima 1942; Costa Lima 1953; Drea \& Gordon 1990; Azeredo et al. 2004), psilídeos (Michaud 2001; Michaud 2004) e afídeos (ARaújo 1940; Bartoszeck 1976; Lucas et al. 1997; Michaud \& BRoWNING 1999; MichaUd 2000).

Vários trabalhos têm sido realizados, relacionando à presença de joaninhas à cultura dos citros (ARAúJo 1940;
Bartoszeck 1976; Bartoszeck 1980, Chagas et al. 1982; Chagas \& Silveira Neto 1985, De Bortoli et al. 2001); Arioli \& Link (1987a) verificaram 23 espécies de coccinelídeos e a preferência destes insetos por esta cultura, mesmo que o alimento preferencial ocorresse em outras plantas (ARIOLI \& LINK, 1987b).

O presente estudo objetivou estudar a ocorrência, flutuação populacional e delinear a distribuição geográfica de coccinelídeos em municípios produtores de citros no Estado do Rio de Janeiro.

\section{Material e Métodos}

Foram realizados levantamentos em quatro regiões do Estado do Rio de Janeiro compreendendo 11 municípios: Baixada Fluminense (Seropédica) e Região das Baixadas Litorâneas (Itaboraí, Tanguá, Rio Bonito, Silva Jardim, Araruama, Iguaba Grande, São Pedro da Aldeia, Cabo Frio, Saquarema e Maricá).

Para o município de Seropédica os levantamentos foram realizados no Campus da Universidade Federal Rural do Rio de Janeiro e no assentamento Moura Costa, no período de janeiro a dezembro de 2000 com periodicidade quinzenal. Na Região Citrícola, foram realizados levantamentos entre dezembro de 2000 a junho de 2001 com periodicidade mensal.

Os levantamentos foram realizados baseandose na metodologia de Perruso \& Cassino (1993), Perruso \& Cassino (1997) e Cassino \& Rodrigues (2004), que se baseaim na presença-ausência dos insetos. Cada planta estudada foi 
previamente dividida imaginariamente em quatro quadrantes, sendo monitoradas 10 plantas por talhão. Para o levantamento dos aleirodídeos e cochonilhas foram observadas 10 folhas por quadrante. Para os inimigos naturais, foram observados todos os quadrante. Foi amostrada uma faixa de cada planta que variou de 1,30 a $1,7 \mathrm{~m}$ os herbívoros quanto para os coccinelídeos (Figura 1). Em cada município foi selecionada uma propriedade para realizar os levantamentos, tendo em vista que mais de uma propriedade inviabilizaria financeiramente o projeto, devido aos custos com transporte e hospedagem, exceto para Seropédica onde foram realizados levantamentos em três propriedades.
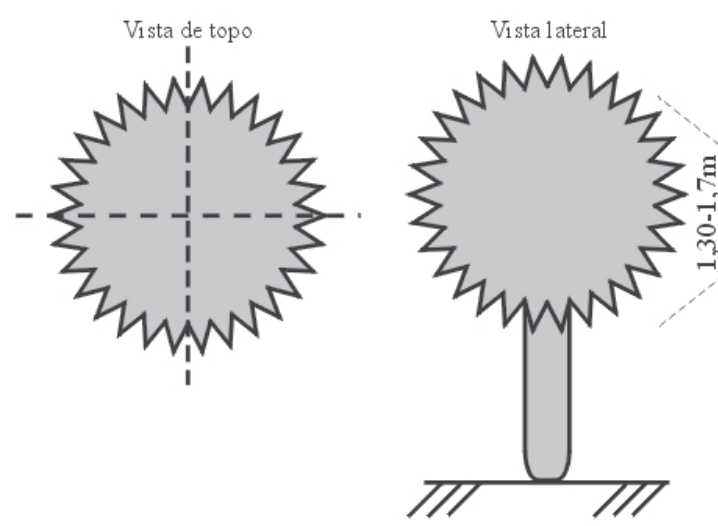

Figura 1. Divisão imaginária de planta para o monitoramento de fitoparasitos e associação entre estes e predadores e formicídeos. Adaptado de CASsino \& Rodrigues (2004).

Para as análises faunísticas da comunidade de coccinelídeos foram utilizados os índices de diversidade de Shanon-Wiener, Margalef, Equitabilidade J (BRower et al. 1997) e dominância de Berger-Parker (BERger \& PARKER 1970), estes índices foram aplicados através do software DivEs - Diversidade de Espécies v2.o (RodRigues 2005).

Os insetos coletados foram submetidos à conservação através da montagem com alfinete entomológico ou acondicionados em tubos de eppendorf de 1,5 mL contendo álcool 70\%. A determinação das espécies foi realizada através da comparação morfológica com o material depositado na Coleção Ângelo Moreira da Costa Lima (CECL), situada no Instituto de Biologia da Universidade Federal Rural do Rio de Janeiro e com auxílio dos trabalhos de Costa Lima $(1941 ; 1953)$, Drea \& Gordon (1990) e Rodrigues (2001).

\section{Resultados e Discussão}

Espécies Encontradas. Os coccinelídeos foram observados em todos os 11 municípios visitados. No período de estudo foram observadas, coletadas e identificadas 11 espécies: A - Azya luteipes Mulsant, 1850; B - Coccidophilus citricola Brèthes, 1905; C - Curinus coeruleus Mulsant, 1850; D - Cycloneda sanguinea (Linnaeus, 1763); E - Hyperaspis notata Mulsant, 1850; F Ladoria desarmata Mulsant, 1850; G - Olla v-nigrum (Mulsant, 1866); H - Pentilia egena (Mulsant, 1850); I - Chilocorus sp.; J Scymnus sp. e L - Stethorus sp. Algumas destas espécies já foram relatadas para o Brasil por GUÉRIN (1953) e associadas à cultura dos citros (ARIOLI \& LiNK 1987a; ARIOLI \& LiNK 1987b), merecendo destaque pela abundância e freqüência com que ocorrem em agroecossistemas cítricos, tornando-se agentes efetivos na regulação da densidade populacional de insetos fitófagos (CHAGAS et al. 1982).

As espécies ocorrem distribuídas da seguinte forma: em Seropédica dez espécies; Araruama, oito; Saquarema e Rio Bonito, cinco; Marica quatro; Silva Jardim e Cabo Frio, três; São Pedro da Aldeia, Iguaba Grande, Tanguá e Itaboraí, duas (Tabela 1, Figura 2).

Os municípios com maior ocorrência de espécies foi Seropédica, devido provavelmente por ser um município com baixa exploração citrícola o que reduz os gastos com defensivos, além das características de exploração da cultura, realizada de forma familiar (agricultura familiar) e um maior período de estudo; e Araruama, devido talvez a maior ocorrência de insetos fitófagos (RoDRIGUEs 2001). As menores ocorrências foram em São Pedro da Aldeia, Iguaba Grande, Tanguá, Itaboraí, (Tabela 1 e Figura 2). Provavelmente a ação antrópica, com a utilização de agroquímicos, que ocasionam a redução dos coccinelídeos, devido à alta vulnerabilidade desses insetos a este tipo de substâncias (OBRYCKI \& KRING 1998; IPERTI 1999; Youn et al. 2003), podendo causar alta mortalidade (100\%) (OlszAK 1999). Entretanto modificações nas práticas agronômicas podem auxiliar na conservação destes insetos (OBRYCKI \& KRING 1998), Como a utilização de produtos seletivos aos inimigos naturais (BARBOSA 2003).

Dentre os coccinelídeos $P$. egena, ocorreu em maior número de municípios (10), seguido por Scymnus sp. (8); A. luteipes (7), L. desarmata (5); C. citricola, C. sanguinea, Chilocorus sp. (3); H. notata, O. v-nigrum, Stethorus sp. (02) e C. coeruleus (01). Esta maior distribuição de P. egena e Scymnus sp. (Tabela 1 e Figura 2), esta relacionada a ampla distribuição de diaspidídeos, principalmente Selenaspidus articulatus (Morgan, 1889), Pinnaspis aspidistrae (Signoret, 1869) e de aleirodídeos Aleurothrixus floccosus Maskell, 1896, Singhiella citrifolii (Morgan, 1893) e Paraleyrodes bondari Peracchi, 1971 (RODRIGUES 2001).

Tabela 1. Distribuição dos coccinelídeos (Coleoptera, Coccinellidae) em 17 Municípios do Estado do Rio de Janeiro, produtores de citros.

\begin{tabular}{|c|c|c|c|c|c|c|c|c|c|c|c|c|}
\hline \multirow[t]{2}{*}{ Município } & \multicolumn{12}{|c|}{ Coccinelídeo* } \\
\hline & $\mathbf{A}$ & B & C & D & $\mathbf{E}$ & $\mathbf{F}$ & G & $\mathbf{H}$ & I & $\mathbf{J}$ & $\mathbf{L}$ & $\Sigma$ \\
\hline Seropédica & $\mathrm{X}$ & $\mathrm{X}$ & & $\mathrm{X}$ & $\mathrm{X}$ & $\mathrm{X}$ & $\mathrm{X}$ & $\mathrm{X}$ & $\mathrm{X}$ & $\mathrm{X}$ & $\mathrm{X}$ & 10 \\
\hline Araruama & $\mathrm{X}$ & & & & $\mathrm{X}$ & $\mathrm{X}$ & $\mathrm{X}$ & $\mathrm{X}$ & $\mathrm{X}$ & $\mathrm{X}$ & $\mathrm{X}$ & 8 \\
\hline Saquarema & $\mathrm{X}$ & & $\mathrm{X}$ & & & $\mathrm{X}$ & & $\mathrm{X}$ & & $\mathrm{X}$ & & 5 \\
\hline Rio Bonito & $\mathrm{X}$ & & & $\mathrm{X}$ & & & & $\mathrm{X}$ & $\mathrm{X}$ & $\mathrm{X}$ & & 5 \\
\hline Marica & $\mathrm{X}$ & $\mathrm{X}$ & & & & $\mathrm{X}$ & & $\mathrm{X}$ & & & & 4 \\
\hline Silva Jardim & $\mathrm{X}$ & $\mathrm{X}$ & & & & $\mathrm{X}$ & & & & & & 3 \\
\hline Cabo Frio & $\mathrm{X}$ & & & $\mathrm{X}$ & & & & $\mathrm{X}$ & & & & 3 \\
\hline S. P. da Aldeia & & & & & & & & $\mathrm{X}$ & & $\mathrm{X}$ & & 2 \\
\hline Iguaba Grande & & & & & & & & $\mathrm{X}$ & & $\mathrm{X}$ & & 2 \\
\hline Tanguá & & & & & & & & $\mathrm{X}$ & & $\mathrm{X}$ & & 2 \\
\hline Itaboraí & & & & & & & & $\mathrm{X}$ & & $\mathrm{X}$ & & 2 \\
\hline$\Sigma$ & 7 & 3 & $\mathbf{1}$ & 3 & 2 & 5 & 2 & 10 & 3 & 8 & 2 & -- \\
\hline
\end{tabular}

${ }^{*} \mathrm{~A}=$ Azya luteipes $; \mathrm{B}=$ Coccidophilus citricola $; \mathrm{C}=$ Curinus coeruleus $; \mathrm{D}=$ Cycloneda sanguinea $\mathrm{E}=$ Hyperaspis notata $; \mathrm{F}=$ Ladoria desarmata $; \mathrm{G}=$ Olla v-nigrum; $\mathrm{H}=$ Pentilia egena; $\mathrm{I}=$ Chilocorus $\mathrm{sp}$; $\mathrm{J}=$ Scymmus sp. e L= Stethorus sp.

No Estado do Rio de Janeiro vem ocorrendo um processo migratório da citricultura, que inicialmente se instalou na Baixada Fluminense e hoje já se encontra na Região Norte e Noroeste do Estado do Rio de Janeiro (VASCONCElos \& VASCONCELOS 2004), levando a crer que juntamente houve uma migração dos insetos fitófagos e seus inimigos naturais, pois com a migração dos coccinelídeos é possível que estes se adaptem facilmente a novas presas (IPERTI 1999), sendo o predador adulto capaz de se deslocar a longas distâncias para localizar o habitat do hospedeiro (VISON 1981 apud GARCia 1991). Além disso, para localizar colônias de afídeos, os coccinelídeos afidófagos são capazes de utilizar o feromônio liberado pelas formigas atendentes, estes predadores também podem ser atraídos por compostos emanados da planta hospedeira da presa, independente de sua presença (KESTEN 1969 apud GARCIA 1991).

Algumas das espécies observadas não são relatadas na literatura, portanto $C$. coeruleus, $H$. notata, L. desarmata e Chilocorus sp. constituem novos registros de ocorrência para a cultura dos citros no Estado do Rio de Janeiro.

Novos estudos devem ser realizados para verificar o potencial destas espécies em programas de controle biológico para a cultura dos citros e a escolha das espécies candidatas 


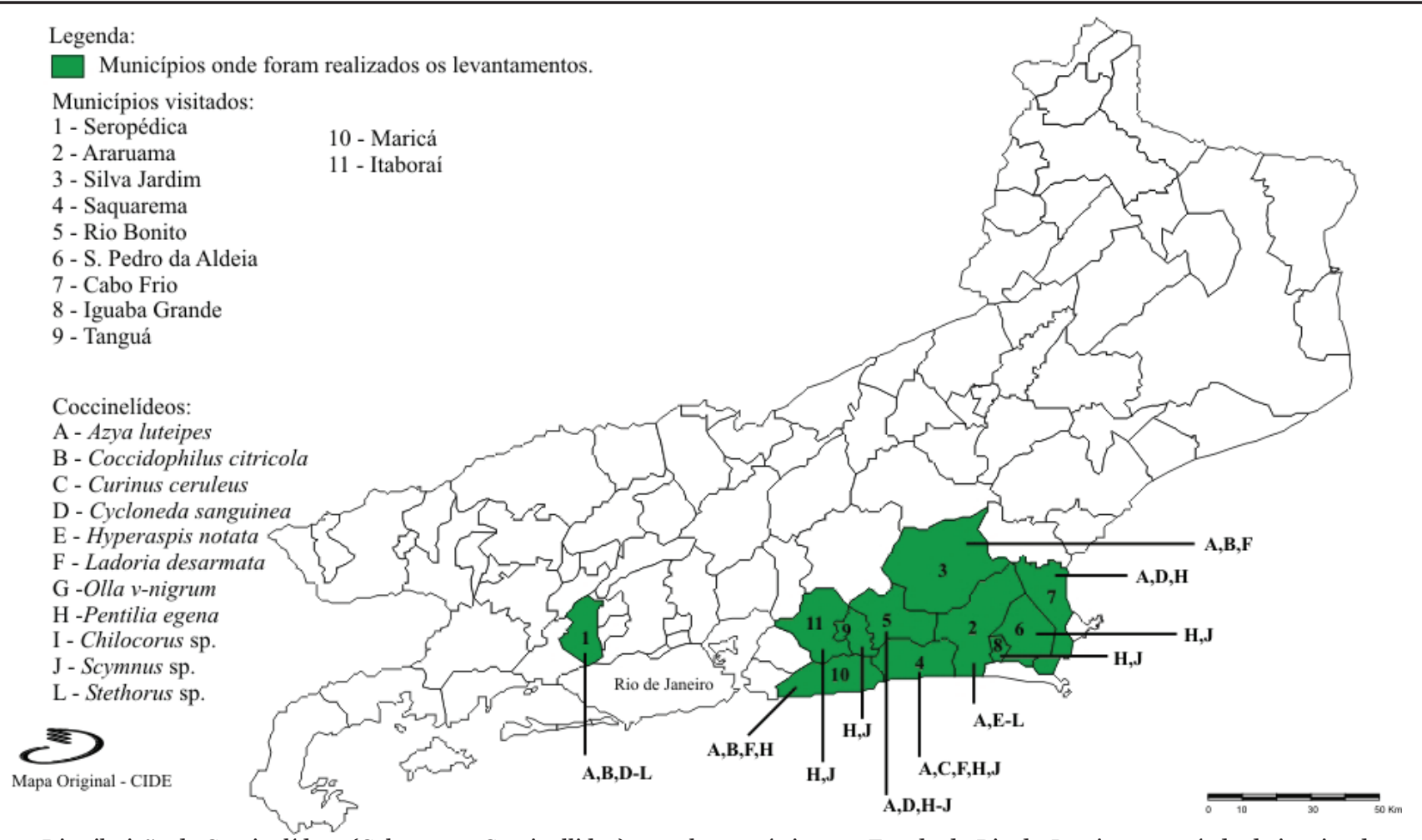

Figura 2. Distribuição de Coccinelídeos (Coleoptera, Coccinellidae), em plantas cítricas no Estado do Rio de Janeiro no período de janeiro de 2000 a junho de 2001.

poderá se dar através dos critérios apresentados por OвRускі \& KRING (1998) e VAN LENTEREN (2000), entretanto alguns aspectos devem ser verificados, como a dificuldade de criação, elaboração de dietas artificiais e manutenção da população no campo (GARCIA 1991).

Flutuação Populacional. Esta parte do estudo somente avaliou o município de Seropédica, por entender que foram realizados monitoramentos durante 12 meses (janeiro a dezembro de 2000), permitindo assim uma avaliação em todas as estações do ano. O coccinelídeo com maior média populacional em todos levantamentos foi $P$. egena (40\%), sendo verificado seu pico populacional em janeiro $(55,8 \%)$ e menor em maio $(28,3 \%)$. Dentre os coccinelídeos considerados mais abundantes ( $P$. egena, A. luteipes, C. sanguinea, O. v-nigrum e H. notata), ou seja, com os maiores índices de presença, $O$. $v$-nigrum e $H$. notata foram as populações que se verificou menor média (8\%) (Figura 3).

Em geral houve um decréscimo populacional da população dos coccinelídeos no decorrer do período de levantamentos, principalmente nos meses mais frios do ano (junho a setembro), exceto para $P$. egena, que apesar do decréscimo de janeiro a maio, houve um aumento populacional nos meses subseqüentes, decaindo novamente em dezembro.

Quando consideradas as espécies menos abundantes, Stethorus sp., obteve maior média em todo período (7\%). Para as demais espécies as médias foram baixas: $C$. citricola, $L$. desarmata e Scymmus sp.(3\%) e Chilocorus sp. (2\%). A flutuação populacional acompanhou a mesma tendência de variação que as espécies abundantes, ou seja, um decréscimo populacional nos meses mais frios, exceto para L. desarmata (Figura 4). Desta forma, mudanças nas condições climáticas e surtos de presas influenciam na biologia e ecologia dos coccinelídeos, entretanto a abundância de presas tem maior influência que as condições climáticas, porém quando estes dois fatores são favoráveis atraem um grande número de coccinelídeos (IPERTI 1999).

Os resultados encontrados por ARIOLI \& LiNK (1987a) incluem uma vasta lista de coccinelídeos e suas respectivas presas e cita que o aumento da população está diretamente ligada com o aumento da população de insetos fitófagos, sendo estes atraídos por suas presas (IPERTI 1999) e seus picos populacionais coincidem com os maiores picos das populações de insetos presas (LINK \& ARIOLI 1986), fato também verificado no presente trabalho.

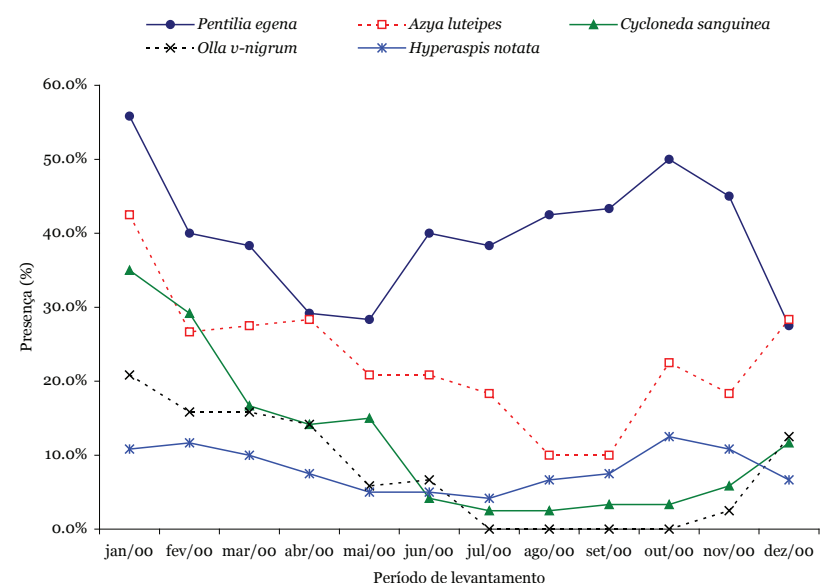

Figura 3. Flutuação populacional dos coccinelídeos (Coleoptera, Coccinellidae) mais abundantes no município de Seropédica, RJ no período de janeiro a dezembro de 2000.

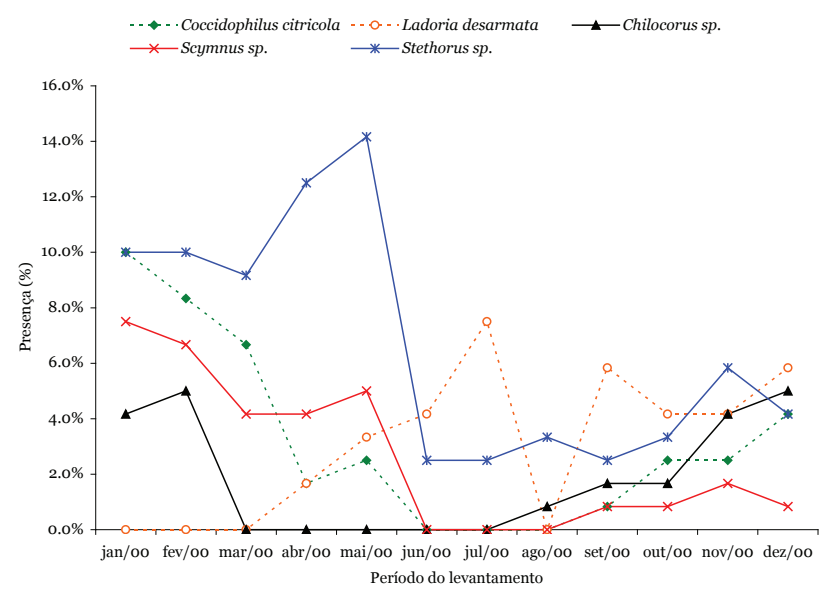

Figura 4. Flutuação populacional dos coccinelídeos (Coleoptera, Coccinellidae) menos abundantes no município de Seropédica, RJ no período de janeiro a dezembro de 2000. 
Diversidade de Espécies. A diversidade em todo período foi considerada alta quando calculada através do índice de Margalef $(\alpha=3,9)$, pois valor semelhante $(\alpha=3,6)$ foi verificado por ARIOLI \& Link (1987a), sendo superior ao valor $(\alpha=1,9)$ encontrado por Chagas \& Silveira Neto (1985). Entretanto a avaliação da diversidade para o tipo de estudo realizado é mais adequada quando se aplica o índice de Shanon-Wiener $\left(\mathrm{H}^{\prime}=0,80\right)$, que segundo BROWER et al. (1997) é um índice mais apropriado para avaliar amostras aleatórias onde há divisão em comunidades, como no presente estudo. Desta forma, a diversidade através do índice H', demonstra que em todo período de estudo houve alta diversidade, pois os valores encontrados nunca foram inferiores a o,5, exceto no mês de agosto $(0,491)$.

A maior diversidade ocorreu em maio $\left(\mathrm{H}^{\prime}=0,827\right)$, não coincidindo com a menor dominância $(0,257)$, ocorrida no mês de abril, por haver dominância de $P$. egena $(\mathrm{d}=0,295)$ e codominância de A. luteipes ( $\mathrm{d}=0,205)$, dando uma falsa impressão de dominância. A maior dominância $(\mathrm{d}=0,646)$ ocorreu em agosto, coincidindo com a menor diversidade $\left(\mathrm{H}^{\prime}=0,491\right) \mathrm{e}$ menor equitabilidade $J(e=0,631)$, esta dominância foi exercida por $P$. egena, fato que ocorreu de janeiro a novembro, sendo que em dezembro a dominância foi exercida por $A$. luteipes $(\mathrm{d}=$ o,276), porém com uma forte co-dominância de $P$. egena $(0,268)$ (Figura 5). Entretanto é de se esperar que a Equitabilidade $\mathrm{J}$ e H' se comportam inversamente à dominância, uma vez que os valores altos destes dois índices indicam uma baixa concentração de dominância (ODum 2001), mas como visto, este fato ocorrerá caso exista apenas uma espécie dominante, pois os modelos matemáticos atuais não conseguem prever a co-dominância de espécie.

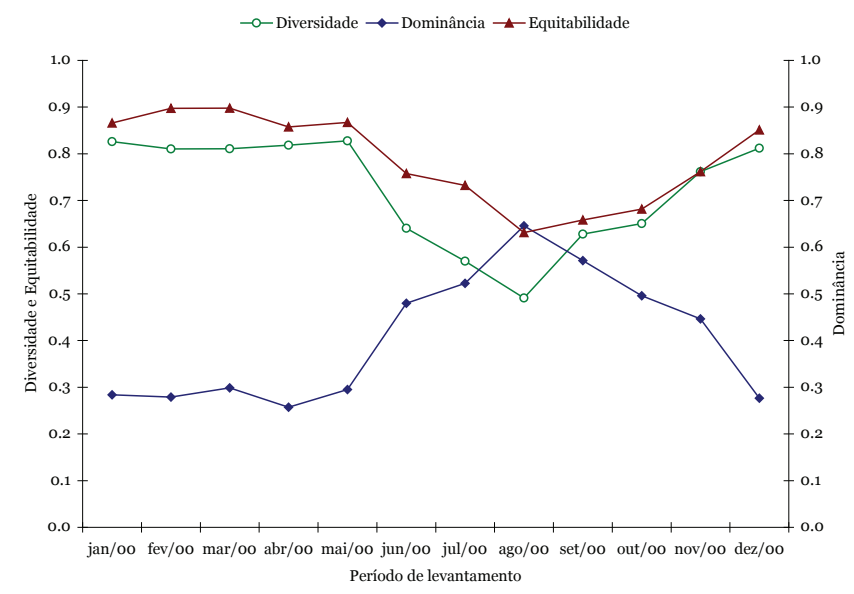

Figura 5. Índices de diversidade (Shanon-Wiener), Equitabilidade $\mathrm{J}$ (e) e dominância (Berger-Parker), da comunidade de coccinelídeos do município de Seropédica, no período de janeiro a dezembro de 2000.

A dominância de $P$. egena e co-dominância de $A$. luteipes, pode ser explicada pela alta infestação de diaspidídeos no período de estudos, sendo a primeira espécie considerada a principal predadora de cochonilhas de carapaças nas condições brasileiras (GRAVENA 1980), sendo ainda listada predando outras cochonilhas (ARIOLI \& LINK 1987a, 1987b).

A diversidade de uma comunidade contribui para redução da dominância (ODum 2001), o que favorece as espécies consideradas raras ou criptas. Assim os coccinelídeos menos abundantes podem exercer um papel importante no agroecossistema cítrico no Estado do Rio de Janeiro.

Estudos relacionados a ecologia e bionomia de inimigos naturais tem importância para os programas de manejo integrado de praga e controle biológico, permitindo o conhecimento a cerca da ocorrência, flutuação populacional e distribuição geográfica, além de determinar quais os possíveis coccinelídeos ser utilizados nestes programas, trazendo aos produtores uma alternativa para o controle dos insetos pragas que ocorrem em seus pomares.

\section{Agradecimentos}

Os autores agradecem ao Prof. Francisco Racca Filho da Universidade Federal Rural do Rio de Janeiro, pela revisão do manuscrito e a Paschoal Coelho Grossi, pelo auxílio na identificação dos coccinelídeos.

\section{REFERÊNCIAS}

Araújo, R.L., 1940. As joaninhas úteis. O Biológico 6: 94-97.

Arioli, M.C.S. \& D. Link, 1987a. Ocorrência de joaninhas em pomares cítricos na Região de Santa Maria, RS. Revista do Centro de Ciências Rurais 17: 213-222.

Arioli, M.C.S. \& D. Link, 1987b. Coccinelídeos da Região de Santa Maria e arredores. Revista do Centro de Ciências Rurais 17: 193-211.

Azeredo, E.H., W.C. Rodrigues, P.C.R. Cassino, 2004. Ocorrência de Selenaspidus articulatus (Morgan) (Hemiptera, Diaspididae) e do predador Pentilia egena (Mulsant) (Coleoptera, Coccinellidae) em Myrtus communis L. (Myrtaceae), em Pinheiral, RJ. Revista Brasileira de Entomologia 48: 569-576.

Barbosa, F.R., R.G. Ferreira, L.H.P. Kiill, E.A. Souza, WW.A. Moreira, J.A Alencar \& F.NP. Haji, 2003. Nível de dano, plantas invasoras hospedeiras, inimigos naturais e controle do psilídeo da goiabeira (Triozoida sp.) no submédio São Francisco. Revista Brasileira de Fruticultura 25: 425-428.

Bartoszeck, A.B., 1976. Afídeos da laranjeira (Citrus sinensis, Osb.) e mimoseira (Citrus reticulata, B.), seus parasitos e predadores. Acta Biologica Paranaense 5: 5-48.

Bartoszeck, A.B., 1980. Ocorrência de Toxoptera citricidus (Aphididae) e seus inimigos naturais em Imperatriz, MA, Brasil, Dusenia 12(1): 9-13.

Berger, W.H. \& F.L., Parker, 1970. Diversity of Planktonic Foraminifera in Deep-Sea sediments. Science, 168: 13451347.

Brower, J. E., J.H. Zar \& C.N. von Ende, 1997. Field and laboratory methods for general ecology. WCB/ McGraw-Hill, 4th, 273p.

Cassino, P.C.R. \& W.C. Rodrigues. 2004. Monitoramento de insetos fitófagos, ácaros, e inimigos naturais, p. 149-157. In: P.C.R. Cassino \& W.C. Rodrigues (coords.). Citricultura Fluminense: principais pragas e seus inimigos naturais. Seropédica, EDUR, 168p.

Chagas, E.F \& S. Silveira Neto, 1985. Uso de coletor de sucção no estudo da entomofauna em um pomar cítrico. Pesquisa Agropecuária Brasileira 20(10): 1125-1141.

Chagas, E.F., S. Silveira Neto, A.J.B.P. Braz, C.P.B. Mateus \& I.P. Coelho, 1982. Flutuação populacional de pragas e predadores em citros. Pesquisa Agropecuária Brasileira 17: 817-824.

Costa Lima, A.M., 1941. Sobre a joaninha Coccidophilus citricola Brèthes, 1905 (Col., Coccinellidae). Revista Brasileira de Biologia 1: 409-414.

Costa Lima, A.M., 1942. Insetos do Brasil - Homópteros. Rio de Janeiro, Escola Nacional de Agronomia, $3^{\circ}$ Tomo, 327p.

Costa Lima, A.M., 1953. Insetos do Brasil - Coleópteros $2^{\mathrm{a}}$ parte. Rio de Janeiro, Escola Nacional de Agronomia, $8^{\circ}$ Tomo, 323p.

De Bortoli, S.A, S.R. Benvenga, S. Gravena \& J.E. Miranda, 2001. Biologia de Pentilia egena Mulsant (Coleoptera: Coccinellidae) e predação sobre Chrysomphalus ficus Ashmead (Homoptera: Diaspididae). Boletin Sanidad Vegetal de Plagas 27: 337-343.

Drea, J.J \& R.D. Gordon, 1990. Coccinellidae, p. 19-40. In: D. Rosen (ed.). Armored Scale Insects: their biology, natural enemies and control. Vol B, New York, Elsevier, xxi+670p.

Garcia, M.A., 1991. Ecologia nutricional de parasitóides e predadores terrestres, p. 289-311. In: A.R. Panizzi \& J.R.P. Parra (eds.). Ecologia nutricional de insetos e suas implicações no manejo de pragas. São Paulo, Manole, 359p.

Gravena, S., 1980. Controle integrado de pragas de pragas dos citros. Citricultura brasileira 2: 648-690. 
Guérin, J., 1953. Coleópteros do Brasil. Soc. Bras. Entomologia. Ed. Fac. Filos. Ciên. Letras, USP, São Paulo, 356p.

Hodek, I., 1973. Biology of Coccinelidae. Academia Publishing House, Prague, 26op.

Iperti, G., 1999. Biodiversity of predaceous coccinellidae in relation to bioindication and economic importance. Agriculture Ecosystems and Environment 74: 323-342.

Katysoyannos, P., K. Ifantis \& D.C. Kontodimas, 1997. Phenology, population trend and natural enemies of Aleurothrixus floccosus (Hom.,: Aleyrodidae) at a newly invaded area in Athens, Greece. Entomophaga 42: 619-628.

Link, D. \& M.C.S. Arioli, 1986. Flutuação populacional de representantes de Coccinellini em culturas de inverno, primavera e verão. Revista do Centro de Ciências Rurais 16: 29-36.

Lucas, E., D. Coderre \& C. Vincent, 1997. Voracity and feeding preferences of two aphidophagous coccinellids on Aphis citricola and Tetranychus urticae. Entomologia Experimentalis et Applicata 85: 151-159.

Michaud, J.P. \& H.W. Browning, 1999. Seasonal abundance of the brown of the brown citrus aphid, Toxoptera citricida, (Homoptera: Aphididae) and its natural enemies in Puerto Rico. Florida Entomologist 82: 424-447.

Michaud, J.P., 2000. Development and reproduction of ladybeetles (Coleoptera: Coccinellidae) on the citrus aphids Aphis spiraecola Patch and Toxoptera citricida (Kirkaldy) (Homoptera: Aphididae). Biological Control 18: 287-297.

Michaud, J.P., 2001. Numerical response of Olla v-nigrum (Coleoptera: Coccinellidae) to infestations of asian citrus psyllid,(Hemiptera: Psylidae) in Florida. Florida Entomologist 84: 608-612.

Michaud, J.P., 2004. Assessment of cotton as an alternative host plant for the brown citrus aphid, Toxoptera citricida (Homoptera: Aphididae). Florida Entomologist 87 105-111.

Obrycki, J.J. \& J.T. Kring, 1998. Predaceous Coccinellidae in Biological Control. Annual Review of Entomology 43: 295321.

Odum, E.P., 2001. Fundamentos de Ecologia. $6^{\mathrm{a}}$ ed. Lisboa: Fund. Calouste Gulbenkian, 927p.

Olszak, R.W., 1999. Influence of some pesticides on mortality and fecundity of the aphidophagous coccinellid Adalia bipunctata L. (Col., Coccinellidae). Journal Applied Entomology 123: 4145.

Peracchi, A.L., 1971. Dois aleirodídeos pragas de Citrus sp. no Brasil (Hom., Aleyrodidae). Arquivos do Museu Nacional 54:145-159.

Perruso, J.C. \& P.C.R. Cassino, 1993. Flutuação populacional de

\section{Como citar este artigo:}

Rodrigues, W.C., P.C.R. Cassino \& R. Silva Filho. 2008. Ocorrência e Distribuição de Coccinelídeos (Coleoptera, Coccinellidae) Associados às Plantas Cítricas no Estado do Rio de Janeiro. EntomoBrasilis, 1(2): 23-27. www.periodico.ebras.bio.br/ojs
Selenaspidus articulatus (Morg.) (Homoptera: Diaspididae) em Citrus sinensis (L.) no Estado do Rio de Janeiro. Anais da Sociedade Entomológica do Brasil 22:401-404.

Perruso, J.C. \& P.C.R. Cassino, 1997. Plano de amostragem presença-ausência para Selenaspidus articulatus (Morg.) (Homoptera: Diaspididae) na cultura da laranja. Anais da Sociedade Entomológica do Brasil 26: 321-326.

Rodrigues, W.C, 2001. Insetos entomófagos de fitoparasitos (Homoptera, Sternorrhyncha) de plantas cítricas no Estado do Rio de Janeiro: ocorrência e distribuição. 9op. Tese (Mestrado em Fitotecnia), Universidade Federal Rural do Rio de Janeiro.

Rodrigues, W.C., 2005. DivEs - Diversidade de Espécies. Versão 2.o. Software e Guia do usuário disponível em: $<$ http://www.ebras.bio.br/dives $>$.

Silva, A.G., C.R. Gonçalves, D.M. Galvão, A.J.L. Gonçalves, J. Gomes, M.N. Silva \& L. Simon, 1968. Quarto catálogo dos insetos que vivem nas plantas do Brasil, seus parasitos e predadores. Ministério da Agricultura, Parte II $1^{\circ}$ Tomo, Rio de Janeiro, 622p.

Sloggett, J.J. \& M.E.N. Majerus, 2000. Habitat preferences and diet in the predatory Coccinellidae (Coleoptera): an evolutionary perspective. Biological Journal of the Linnean Society 70: 63-88.

Ulusoy, M.R., G. Vatansever, L. Erkiliç \& N. Uygun, 2003. Studies on Aleurothrixus floccosus (Maskell) (Homoptera, Aleyrodidae) and its parasitoid, Cales noacki Howard (Hymenoptera, Aphelinidae) in the East Mediterranean Region of Turkey. Journal Pest Science 76: 163-169.

Van Lenteren, J.C., 2000. Critérios de seleção para avaliação de inimigos naturais em controle biológico, p 1-19. In: V.H. P Bueno (ed.). Controle biológico de pragas: produção massal e controle de qualidade. Lavras, UFLA, 207p.

Vasconcelos, H.O. \& M.A.S. Vasconcelos, 2004. Histórico da citricultura do Estado do Rio de Janeiro, p. 1-7 In: P.C.R. Cassino \& W.C. Rodrigues (coords.). Citricultura Fluminense: principais pragas e seus inimigos naturais. Seropédica, EDUR, 168p.

Youn, Y.N., M.J. Seo, J.G. Shin, C. Jang \& Y.M. Yub, 2003. Toxicity of greenhouse pesticides to multicolored Asian lady beetles, Harmonia axyridis (Coleoptera: Coccinellidae). Biological Control 28: 164-170.

Recebido em: 16/03/2008

Aceito em: 06/06/2008

$$
* * * * * * * * * * * * *
$$
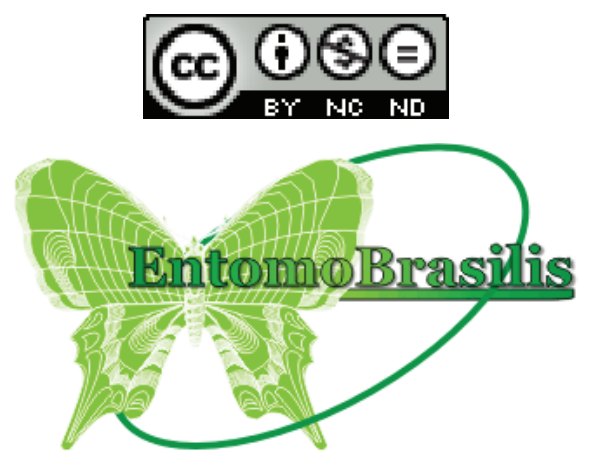


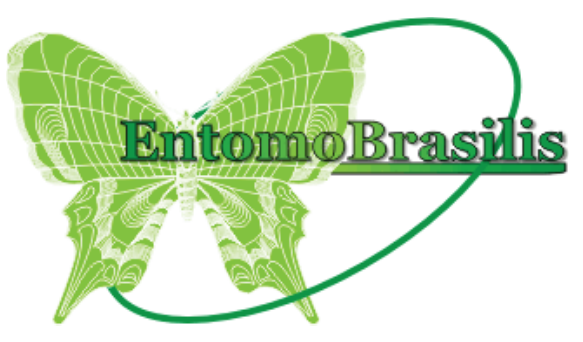

